Preoperative plasma TIMP-1 is an independent prognostic indicator in patients with primary colorectal cancer

\title{
a prospective validation study
}

Birgisson, Helgi ; Nielsen, Hans J.; Christensen, Ib Jarle; Glimelius, Bengt ; Brunner, Nils

Published in:

European Journal of Cancer

DOI:

10.1016/j.ejca.2010.06.009

Publication date:

2010

Document version

Early version, also known as pre-print

Citation for published version (APA):

Birgisson, H., Nielsen, H. J., Christensen, I. J., Glimelius, B., \& Brunner, N. (2010). Preoperative plasma TIMP-1 is an independent prognostic indicator in patients with primary colorectal cancer: a prospective validation study. European Journal of Cancer, 46(18), 3323 -3331. https://doi.org/10.1016/j.ejca.2010.06.009 


\title{
Preoperative plasma TIMP-1 is an independent prognostic indicator in patients with primary colorectal cancer: A prospective validation study
}

\author{
Helgi Birgisson ${ }^{a, *}$, Hans J. Nielsen ${ }^{b}$, Ib Jarle Christensen ' , Bengt Glimelius ${ }^{d, e}$, \\ Nils Brünner $f$ \\ a Department of Surgery, Uppsala University, 75185 Uppsala, Sweden \\ ${ }^{\mathrm{b}}$ Department of Surgical Gastroenterology, Huidoure Hospital, University of Copenhagen, Hvidoure, Denmark \\ c Finsen Laboratory, Rigshospitalet, Copenhagen, Denmark \\ d Department of Oncology, Radiology and Clinical Immunology, Uppsala University, Uppsala, Sweden \\ e Department of Oncology and Pathology, Karolinska Institute, Stockholm, Sweden \\ ${ }^{f}$ Section of Pathobiology, Department of Veterinary Disease Biology, Faculty of Life Sciences, University of Copenhagen, Copenhagen, Denmark
}

\section{A R T I C L E I N F O}

Article history:

Received 15 April 2010

Received in revised form 3 June 2010

Accepted 4 June 2010

Available online 7 July 2010

\section{Keywords:}

Colorectal cancer

Surgery

TIMP-1

CEA

Survival

Prognosis

\begin{abstract}
A B S T R A C T
Background: Previous studies have suggested plasma tissue inhibitor of metalloproteinases1 (TIMP-1) as a stage independent prognostic marker in colorectal cancer (CRC) patients. The aim was to validate plasma TIMP-1 and serum carcino-embryonic antigen (CEA) levels as prognostic indicators in an independent population-based cohort of patients with CRC. Patients and methods: During 2000-2003, plasma and serum were collected preoperatively from 322 patients treated for primary CRC. TIMP-1 and CEA levels were determined by validated ELISA platforms.

Results: High TIMP-1 and CEA levels each associated with poor overall survival (OS); TIMP-1 (hazard ratio (HR) 2.1; 95\% confidence interval (CI) 1.6-2.7) and CEA (HR 1.2; 95\% CI 1.1-1.3), and disease-free survival (DFS); TIMP-1 (HR 2.0; 95\% CI: 1.5-2.6) and CEA (HR 1.2; 95\% CI: 1.11.4) in univariate analyses. In stratified analyses of stages II and III, TIMP-1 levels associated significantly with OS and DFS in stages II and III, associations were not found for CEA. Multivariate analysis for OS, including TIMP-1 and CEA levels and clinico-pathological baseline variables, revealed significant association of TIMP-1 (HR 1.8; 95\% CI 1.3-2.4) but not CEA levels.

Conclusions: This independent prospective validation study confirms the significant association between preoperative plasma TIMP-1 levels and survival of CRC patients: TIMP-1 provided stronger prognostic information than CEA. Thus, this study brings plasma TIMP-1 to the next level of evidence for its clinical use as a prognostic marker in CRC patients.
\end{abstract}

(c) 2010 Elsevier Ltd. All rights reserved.

\section{Introduction}

Colorectal cancer (CRC) is the most common cancer in Europe and ranks second as the cause of cancer-related deaths in developed countries. ${ }^{1}$ Although survival has improved, almost half of the patients with CRC are expected to die from their disease. About one-fifth of patients have non-curable metastatic disease at diagnosis and for those with curable

\footnotetext{
* Corresponding author: Tel.: +46 186110000; fax: +46 21175860 .

E-mail address: helgi.birgisson@surgsci.uu.se (H. Birgisson). 
disease, one-third will develop recurrent disease leading to death, despite the most modern surgical and oncological treatments.

Major expectations are laid on biomarkers for providing more precise prognostic information than the current tumour, lymph node and metastasis (TNM) staging system. Carcino-embryonic antigen (CEA), which has been studied as a marker of CRC since the 1960s, is the only soluble biomarker recommended for use in CRC, being recommended primarily for monitoring after primary surgery. ${ }^{2}$ As yet, no serological biomarkers have obtained sufficient evidence for routine clinical use in identifying poor prognosis stage II CRC patients, who might be candidates for adjuvant treatment, or good prognosis stage III patients, who could be saved from adjuvant chemotherapy or receive a more mild form for adjuvant treatment other than the oxaliplatin-based treatment generally used for stage III disease. The latest European Group on Tumour Markers (EGTM) recommendations failed to identify additional biomarkers for separating stage II CRC patients into different prognostic groups. ${ }^{3}$ However, the EGTM proposes tissue inhibitor of metalloproteinases-1 (TIMP-1) as a promising new prognostic biomarker, although TIMP-1 needs to be validated before any conclusion on its use can be drawn.

TIMP-1 regulates the activity of matrix metalloproteinases (MMP), which are enzymes involved in degradation of the extracellular matrix (ECM), and thus, may be involved in cancer invasion and metastasis. ${ }^{4}$ CRC patients have higher levels of plasma TIMP-1 than healthy controls, ${ }^{5-7}$ whereas, patients with premalignant adenomas have similar plasma TIMP-1 levels as those of the healthy people. ${ }^{8}$ Furthermore, high preoperative plasma TIMP-1 levels are associated with poor prognosis in CRC, independent of stage, ${ }^{9,10}$ an observation also seen for high postoperative levels of TIMP-1. ${ }^{11}$ If plasma TIMP-1 can be used to reliably identify stage II and stage III CRC patients in whom adjuvant treatment is indicated and whether it should be single-drug 5FU or an oxaliplatin-based combination, it may have high potential for guiding individualised treatment of these patient groups. However, the use of plasma TIMP-1 as a prognostic parameter in CRC requires that the previous results be validated independently with patients and patient samples external to the original institutions. In this context, there are research groups that have already confirmed the association of high plasma TIMP-1 levels with poor prognosis in CRC. ${ }^{12-14}$ In a medical society where TNM staging of CRC patients has improved with the help of CT and MRI scans and where better surgical and oncological treatments are offered, together with improved pathological examination, it is necessary to continue evaluation of promising biomarkers such as TIMP-1 on a current CRC cohort.

Therefore, the aim was to validate the plasma TIMP-1 and the serum CEA levels as prognostic indicators in a populationbased cohort of patients with CRC.

\section{Patients and methods}

\subsection{Patients}

The study was prospective and the cohort was population based, including patients treated for CRC at the Department of Surgery, Central District Hospital, Västerås, County of Väst- manland, Sweden, which has 260,000 inhabitants in the region. The study period was between August 2000 and December 2003 and the inclusion criterion was histologically verified adenocarcinoma of the rectum or colon. Acceptance to participate and to provide blood samples for tumour marker studies was by informed consent.

Information on tumour stage, microscopic resection margins, grade of differentiation and vascular and neural invasion was retrieved from pathology reports.

In the Regional Oncology Registry, 415 patients were treated for CRC in the county of Västmanland during this period. From these patients, 322 patients (stages I-IV) were included in the study, of which 271 had curative resections. The main reasons for non-inclusion $(n=93)$ were acute operation $(n=32)$, patients who were not able or did not want to participate in the study $(n=19)$, non-admission to the hospital because of palliative care $(n=8)$, cancer in a polyp $(n=7)$ and for 27 patients the reason for non-inclusion was unknown. Information on cancer recurrence, death and cause of death of the study cohort was sampled by matching with the Regional Oncology Registry and hospital records.

\subsection{Blood sampling}

The day before the planned resection of the CRC, blood samples were drawn preoperatively into endotoxin-free collection tubes with EDTA as an anticoagulation agent for plasma. Tubes without anticoagulation agent were used for serum. The blood samples were processed for plasma and serum by centrifugation at $2000 \mathrm{~g}$ for $10 \mathrm{~min}$ at room temperature. The samples were stored at $-70^{\circ} \mathrm{C}$ until analysed for TIMP-1 and CEA in 2008.

\subsection{Timp-1}

All assays were performed without knowledge of the clinicopathological data. A thoroughly validated ELISA was used to measure TIMP-1 levels in plasma. ${ }^{15}$ In brief, an affinity-purified sheep polyclonal anti-TIMP-1 antibody was used to capture TIMP-1 in the specimens. MAC15, a murine monoclonal antiTIMP-1 IgG1, was used to detect the TIMP-1: the MAC15 antibody recognises both free and MMP-bound TIMP-1, therefore, the assay can be considered as a total TIMP-1 assay. A rabbit anti-mouse immunoglobin/alkaline phosphatase conjugate (No. D0314, DAKO, Glostrup, Denmark) was used for kinetic rate measurements, which were collected every 10 min over a $1 \mathrm{~h}$ period. KinetiCalc II software (Bio-Tek Instruments, Winooski, VT) was used to calculate the TIMP-1 levels in the specimens. Before performing the assay, all specimens were thawed at $37^{\circ} \mathrm{C}$. On each plate, four reference plasma samples were included to ensure optimal performance of the assay on each assay run. The intra- and inter-assay variations were below $10 \%$.

\subsection{CEA}

The CEA determination was on serum with a commercially available MTPL-EIA kit (RE59101) (IBL; Immuno Biological Laboratories; http://www.ibl-hamburg.com). The analysis is based on the principle of a Solid-Phase-Enzyme-Linked immunosorbent assay. According to the manufacturer's 
instructions, this assay has a detection limit of $1 \mathrm{ng} / \mathrm{ml}$ and the standard range is $5-75 \mathrm{ng} / \mathrm{ml}$.

\subsection{Endpoints}

Endpoints were defined according to the recommendations of Punt and colleagues. ${ }^{16}$ Overall survival (OS) was measured from the date of surgery to the date of death from all causes. Cancer-specific survival (CSS) was measured from the date of surgery to the date of death by CRC; the observations were censored at death from causes other than CRC and at the end of the study period. Curatively resected patients were defined as all patients included with stages I-III disease and microscopically free resection margins (R0). For the curatively resected patients, disease-free survival (DFS) was calculated and measured from the date of surgery to the date of diagnosis of a local/distal recurrence, second primary CRC/non-CRC, or the date of death due to cancer. Time to recurrence (TTR) was calculated and measured from the date of surgery to the date of diagnosis of a local/distant recurrence or to the date of death due to CRC and censored at the date of death due to reasons other than CRC. A second primary CRC/nonCRC was not regarded as recurrence. All observations were censored at the end of the study period (15th November 2008). The recommendations by REMARK ${ }^{17}$ were followed.

\subsection{Statistical methodology}

Descriptive statistics are presented by frequencies and percentages for categorical data and by the median and range (quartiles as well) for continuous variables. Spearman rank correlation coefficient was used as a measure of association and tests for location were with the Wilcoxon rank sum test. The Kaplan-Meier method was used to estimate survival probabilities, and the log-rank test was used to test for equality between strata grouping TIMP-1 and CEA by their respective tertiles for graphical presentation. The Cox proportional hazards model was applied for univariate analyses and for multivariate analyses of OS and DFS. The levels of TIMP-1 and CEA were $\log$ transformed $\left(\log _{2}\right.$ this means a twofold difference in the marker level corresponded to the estimated hazard ratio (HR)) and treated as continuous variables for the uni- and multivariable analyses of OS and DFS. The assumption of the proportional hazards model and the linearity were validated graphically and by examination of the residuals. The significance level was set to $5 \%$. The SAS ${ }^{\circledR}$ software package (version 9.1; SAS Institute, Cary, NC, USA) was used to manage patient data and to perform all statistical analyses.

Note: The resulting multivariate estimates could be used to define clinically relevant cutpoints: these would be adjusted for age, gender and other potential confounders.

Ethical approval (no. 00-001) was obtained from the Ethics Committee at Uppsala University, Uppsala, Sweden.

\section{Results}

\subsection{Patient characteristics}

The total number of patients included in the study was 322 and the median observation time for surviving patients was
6.5 years (range 4.9-8.2 years). Major clinico-pathological characteristics and marker levels of the cohort are presented in Table 1 . The median age was 73 years (range 34-94 years) and the median number of lymph nodes analysed were 15 (range 1-55). Two older patients with rectal cancer and one with colon cancer in a polyp had a local excision of the tumour and one patient with locally advanced colon cancer was treated with radio-chemotherapy only; therefore, these patients did not have a definitive disease stage (Table 1). Six patients with colon cancer and none with rectal cancer underwent acute resection. Among the 322 included patients, 271 patients had curative resection. Preoperative radiation therapy was used in 79 rectal cancer patients, three received chemoradiation therapy and one (stage IV) neoadjuvant chemotherapy only. One patient with distal sigmoid colonic cancer received preoperative radiation therapy and one colonic cancer received neoadjuvant chemotherapy. Adjuvant chemotherapy was given in 71 colon and 34 rectal cancer patients, mainly to those under 75 years with stage III disease.

Fifty-five curatively resected patients developed cancer recurrence $(20 \%)$, one patient had local recurrence and distant metastasis, two had local recurrence only, and 52 had distant metastases only. For the curatively resected patients, one recurrence was observed in two patients treated with only local excision, one (2\%) in the 44 patients with stage I, 17 (13\%) in the 133 patients stage II patients and 36 (39\%) in the 92 patients with stage III.

\begin{tabular}{|c|c|c|}
\hline Variable & Label & $n$ \\
\hline Gender & $\begin{array}{l}\text { Male } \\
\text { Female }\end{array}$ & $\begin{array}{l}163 \\
159\end{array}$ \\
\hline Localisation & $\begin{array}{l}\text { Colon } \\
\text { Rectum }\end{array}$ & $\begin{array}{l}216 \\
106\end{array}$ \\
\hline Disease stage & $\begin{array}{l}\text { I } \\
\text { II } \\
\text { III } \\
\text { IV } \\
\text { Unknown }\end{array}$ & $\begin{array}{r}44 \\
134 \\
94 \\
46 \\
4\end{array}$ \\
\hline Differentiation grade & $\begin{array}{l}\text { Well-moderate } \\
\text { Poor } \\
\text { Unknown }\end{array}$ & $\begin{array}{r}249 \\
70 \\
3\end{array}$ \\
\hline Vascular invasion & $\begin{array}{l}\text { Present } \\
\text { Not present } \\
\text { Unknown }\end{array}$ & $\begin{array}{r}38 \\
276 \\
8\end{array}$ \\
\hline Neural invasion & $\begin{array}{l}\text { Present } \\
\text { Not present } \\
\text { Unknown }\end{array}$ & $\begin{array}{r}8 \\
306 \\
8\end{array}$ \\
\hline Mucinous & $\begin{array}{l}\text { Present } \\
\text { Not present } \\
\text { Unknown }\end{array}$ & $\begin{array}{r}43 \\
271 \\
8\end{array}$ \\
\hline Number of lymph nodes analysed & $\begin{array}{l}<12 \\
\geqslant 12 \\
\text { Unknown }\end{array}$ & $\begin{array}{r}95 \\
224 \\
3\end{array}$ \\
\hline
\end{tabular}




\subsection{Tumour marker levels}

CEA levels correlated with disease stage $(P<0.01)$ and curative resection $(P<0.01)$, and marginally with vascular invasion $(P=0.04)$, with higher levels seen in advanced disease stages, non-curatively treated patients and in patients where vascular invasion was seen in the tumour. No significant associations were found for localisation, differentiation grade and neural invasion. TIMP-1 associated marginally with curative resection $(P=0.05)$, but not with any of the other clinicopathological variables. Increased age correlated with high TIMP-1 levels $(P<0.01)$ in Spearman rank correlation: an association that was not seen for CEA levels $(P=0.22)$. Gender was not associated with TIMP-1 or CEA levels or age. For the whole cohort, the median TIMP-1 level was $156 \mathrm{ng} / \mathrm{ml}$ (range 57-613) and the median CEA level was $2.7 \mathrm{ng} / \mathrm{ml}$ (range 02000).

TIMP-1 and CEA levels were interrelated with high levels of TIMP-1 seen in patients with high levels of CEA, also seen in each subgroup of disease stage.

\subsection{Survival analysis}

The number of events was 147 (46\%) for OS, 80 (25\%) for CSS, 125 (46\%) for DFS and 55 (20\%) for TTR. In the total population $(n=322)$, high TIMP-1 and CEA levels treated as continuous variables were associated with poorer OS, CSS and DFS in univariate COX model analyses, with TIMP-1 levels more significantly associated than CEA (Table 2). Increased CEA levels associated marginally with shorter TTR, a correlation not shown for TIMP-1.

Tertiles were used to divide TIMP-1 and CEA levels in the Kaplan-Meier estimates of survival probabilities and the log-rank test was used for comparing strata. These plots are primarily graphical illustrations and the cutpoints should not be interpreted otherwise. Increased TIMP-1 and CEA levels revealed significant association with poor OS (Fig. 1a and b). Stratifying the data into disease stages II and III revealed a significant association of increased TIMP-1 levels with poorer OS in patients with CRC stages II $(P=0.02)$ and III $(P<0.01)$ (Fig. 1c and d): a correlation that could not be shown for CEA ( $P=0.1$ and $P=0.13$ ) (Fig. 1e and $f$ ).

A similar analysis with DFS as endpoint revealed significant association of high TIMP-1 levels with poor DFS $(P<0.01)$ (Fig. 2a). These correlations were also significant in stratified analyses of stage II $(P<0.01)$ (Fig. $2 b$; Table 3$)$ and stage III $(P<0.01)$ (Fig. 2c; Table 3). CEA levels were not significantly correlated with DFS in these analyses (Table 3).

Multivariate Cox regression analysis for OS, including the TIMP-1 and CEA levels and clinico-pathological baseline variables, for all 322 patients revealed significant association of TIMP-1 $(P<0.01)$ but not CEA $(P=0.20)$ (Table 4$)$. Other significant variables were age, disease stage, and postoperative chemotherapy.

The same multivariate model was used for the curatively resected patients $(n=271)$. This analysis revealed the same individual relationship of high TIMP-1 with poor OS (HR 1.9; 95\% CI 1.4-2.7), with age and disease stage as other individual significant parameters.

\subsection{Time to recurrence}

Stage III patients were stratified into four groups based on age and gender; adjusted CEA and TIMP-1 levels; and, whether adjuvant chemotherapy was given, or not. The analysis revealed significant differences in TTR, with those having high TIMP-1 and/or CEA and not receiving adjuvant treatment having the highest risk for recurrence, and those with low TIMP-1 and/or low CEA and treated with adjuvant chemotherapy having the lowest risk for recurrence (Fig. 3). However, these interactions were not significant $(P=0.20)$ during multivariate analysis including lymph node (N) stage (N1 versus $\mathrm{N} 2$ ).

\section{Discussion}

This prospective study confirmed the significant associations between high preoperative plasma TIMP-1 levels and poor survival of patients with primary CRC. These associations were observed for both DFS and OS analyses of the entire cohort, in the stratified analyses of stages II and III disease and in the multivariate analyses including conventional clinicopathological baseline parameters.

Thus, this study can be considered as an independent prospective validation study that confirmed our previous results on TIMP-1 in the prognostic setting of CRC, i.e. previous results have consistently ascertained ${ }^{9-11,18}$ that increased plasma TIMP-1 levels are significantly associated with poor prognosis:, this is also supported by other groups. ${ }^{12-14}$

The basis of present prognostic estimation and prediction of recurrence in patients with CRC is the TNM staging system. ${ }^{19}$ Curative treatment of CRC can only be achieved with a resection of the primary tumour and eventual metastases.

\begin{tabular}{|c|c|c|c|c|c|c|c|}
\hline \multirow[t]{2}{*}{ Endpoint } & \multirow[t]{2}{*}{$n$} & \multicolumn{3}{|c|}{ TIMP-1 } & \multicolumn{3}{|c|}{ CEA } \\
\hline & & HR & $95 \%$ CI & $P$-value & HR & $95 \%$ CI & $P$-value \\
\hline OS & 322 & 2.1 & $1.6-2.7$ & $<0.01$ & 1.2 & $1.1-1.30$ & $<0.01$ \\
\hline CSS & 322 & 1.9 & $1.3-2.6$ & $<0.01$ & 1.3 & $1.2-1.4$ & $<0.01$ \\
\hline DFS & 271 & 2.0 & $1.5-2.6$ & $<0.01$ & 1.2 & $1.1-1.4$ & $<0.01$ \\
\hline TTR & 271 & 1.4 & $0.95-2.1$ & 0.09 & 1.2 & $1.1-1.3$ & 0.03 \\
\hline
\end{tabular}

TIMP-1: tissue inhibitor of metalloproteinases 1; CEA: carcino-embryonic antigen; OS: overall survival; CSS: cancer specific survival; DFS: disease-free survival; TTR: time to recurrence; HR: hazard ratio; and CI: confidence interval. 
a

TIMP-1 OS

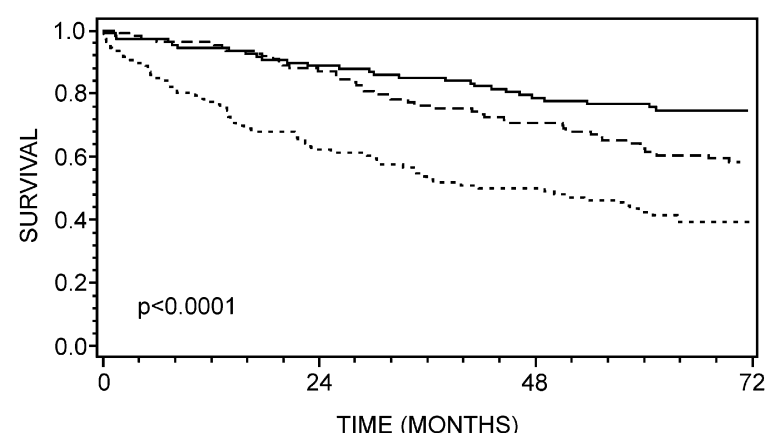

EVENTS PATIENTSAT RISK

$\begin{array}{rrrrrrrr}30 & 107 & 101 & 95 & 91 & 84 & 78 & -1 \\ 48 & 109 & 105 & 95 & 83 & 77 & 66 & \cdots \\ 69 & 106 & 82 & 66 & 56 & 53 & 43 & \cdots \cdots \cdots\end{array}$

C

TIMP-1 OS Stage II

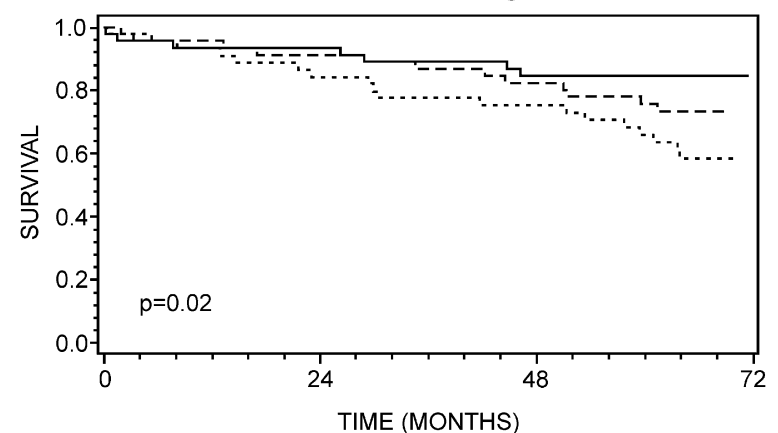

EVENTS PATIENTS AT RISK

$\begin{array}{rllllllll}8 & 45 & 42 & 42 & 40 & 38 & 35 & -1 \\ 13 & 45 & 43 & 41 & 39 & 37 & 33 & \cdots \\ 20 & 44 & 41 & 37 & 34 & 33 & 27 & \cdots \cdots \cdots & 3\end{array}$

e

CEA OS Stage II

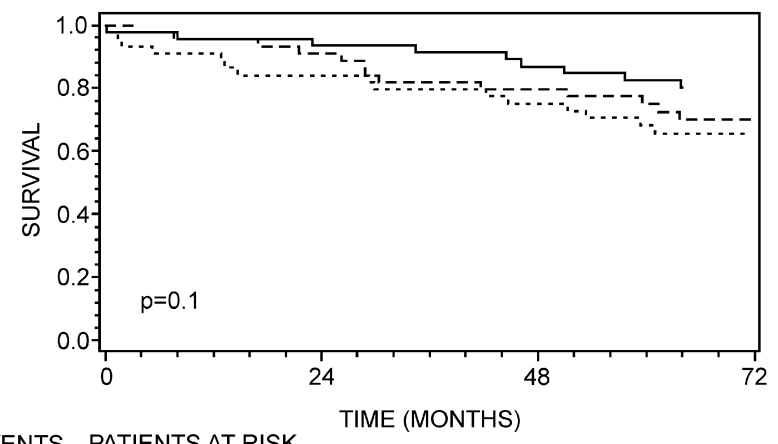

EVENTS PATIENTS AT RISK

$\begin{array}{rrrrrrrr}8 & 46 & 44 & 43 & 42 & 40 & 35-\ldots & 1 \\ 13 & 44 & 42 & 40 & 36 & 35 & 32 & -\ldots . . \\ 20 & 44 & 40 & 37 & 35 & 33 & 28 & \ldots \ldots \ldots\end{array}$

b

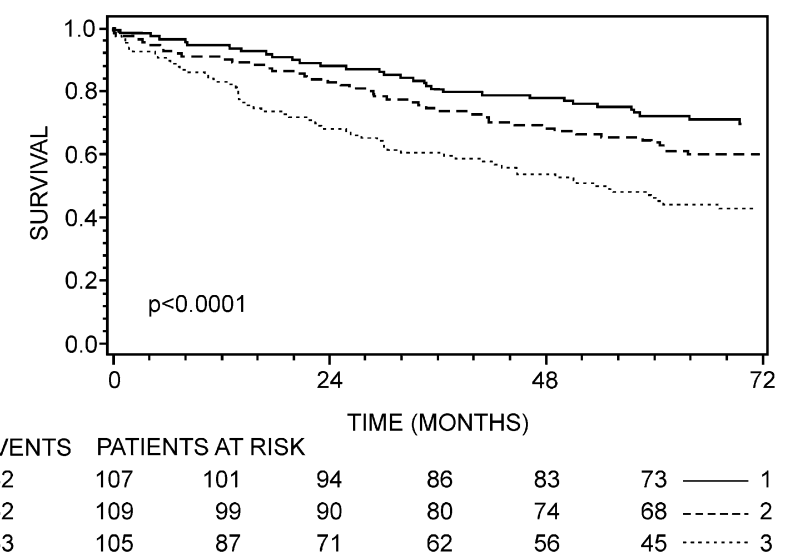

d

TIMP-1 OS Stage III

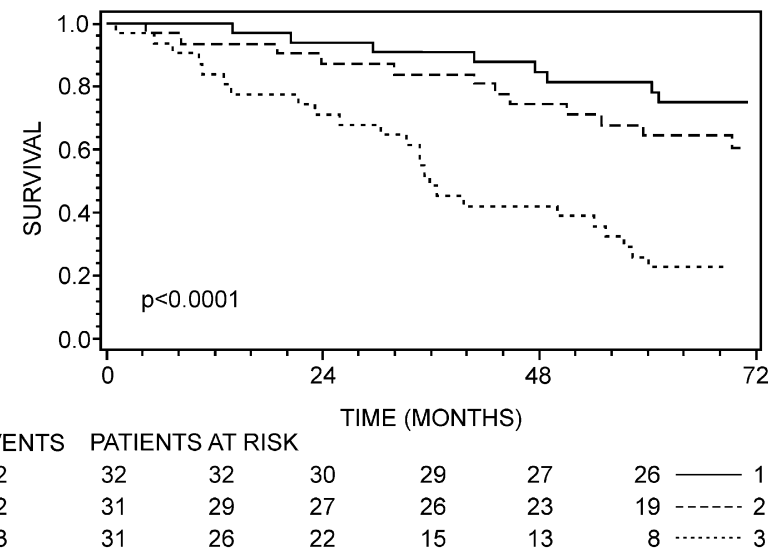

f

CEA OS Stage III

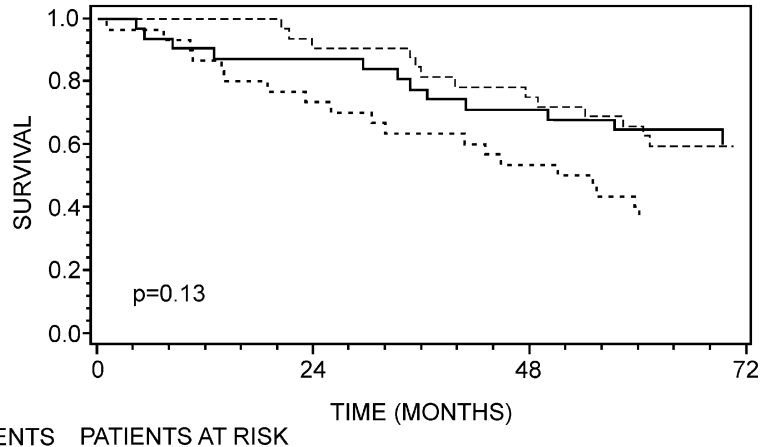

$\begin{array}{llllllll}12 & 31 & 28 & 27 & 24 & 22 & 20-1 \\ 18 & 32 & 32 & 29 & 26 & 24 & 20-\cdots \\ 19 & 30 & 26 & 22 & 19 & 16 & 12 \ldots \ldots \ldots 3\end{array}$

Fig. 1 - Kaplan-Meier estimates of overall survival stratified by TIMP-1 and CEA levels with the respective tertiles as cutpoints. (a) TIMP-1 all patients; (b) CEA all patients; (c) TIMP-1 stage II; (d) TIMP-1 stage III; (e) CEA stage II; AND (f) CEA stage III. The number of patients at risk at $0,12,24,36,48$ and 60 months are shown below the axis and the number of events (deaths) to the left. P-values are for the log-rank statistic. TIMP-1: tissue inhibitor of metalloproteinase 1; CEA: carcino-embryonic antigen and OS: overall survival.

Based on disease stage, CRC patients are selected for surgery alone, preoperative radiation or radio-chemotherapy in case of rectal cancer, adjuvant single-drug or combination chemotherapy in colon cancer or palliative chemotherapy in advanced cancers. The aim of modern cancer treatment is to individualise therapy through the identification of patients with high recurrence risks and offer the patients neoadjuvant or more intense adjuvant therapy, while omitting these treatments in patients with low risk of recurrence. Such procedures would improve patient survival and quality of life by reducing the probability of adverse effects and optimise the use of health care resources. 
a

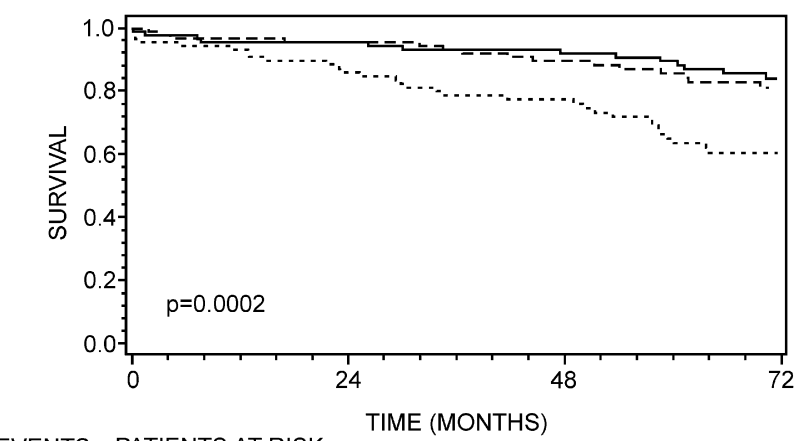

b

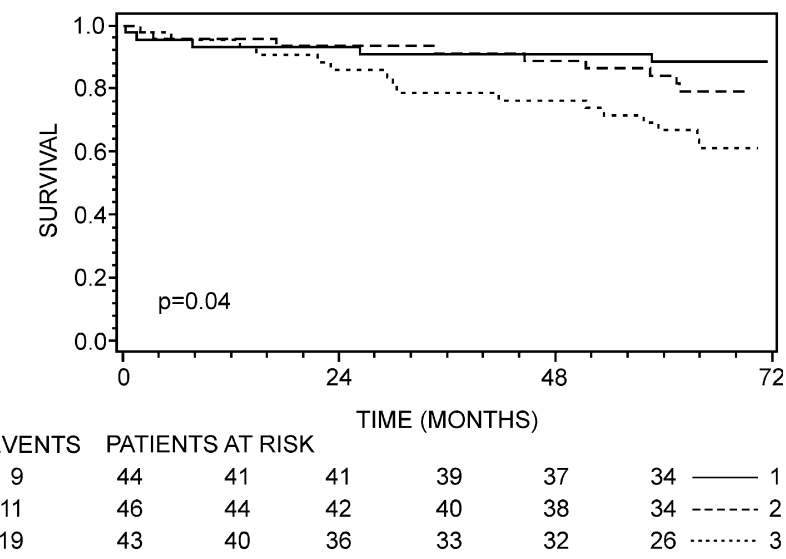

C TIMP-1 DFS Stage III

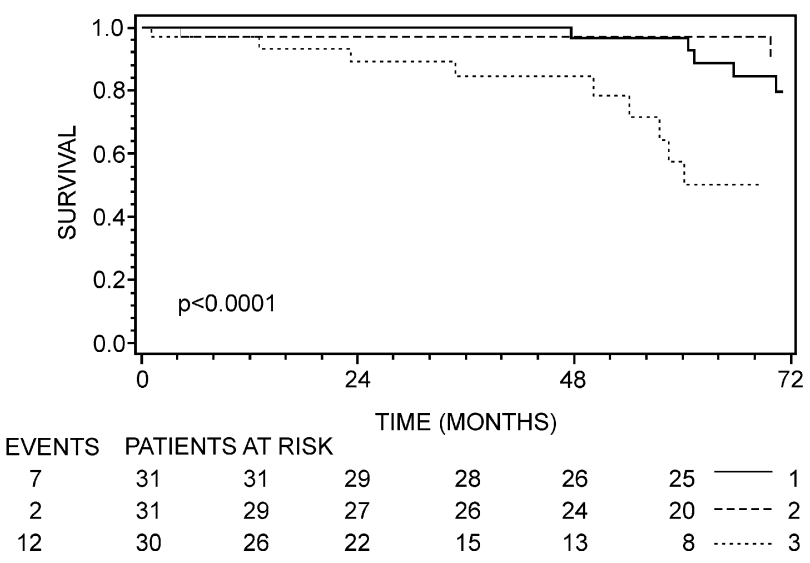

Fig. 2 - Kaplan-Meier estimates of disease-free survival in curatively treated patients with colorectal cancer, stratified by TIMP-1 levels with the tertiles as cutpoints. (a) All curatively treated patients; (b) disease stage II and (c) disease stage III. Pvalue is for the log-rank statistic. The number of patients at risk at $0,12,24,36,48$ and 60 months are shown below the axis and the number of events to the left. TIMP-1: tissue inhibitor of metalloproteinase 1; CEA: carcino-embryonic antigen and DFS: disease-free survival.

\section{Table 3 - Univarate Cox regression analyses for OS and DFS for TIMP-1, and CEA stratified for patients with disease stages I} and III colorectal cancer.

\begin{tabular}{lrrrrrrrr} 
Endpoint & $n$ & \multicolumn{3}{c}{ TIMP-1 } & & \multicolumn{3}{c}{ CEA } \\
\cline { 3 - 5 } & & HR & $95 \%$ CI & P-value & & HR & $95 \%$ CI & P-value \\
\hline OS stage II & 134 & 2.3 & $1.5-3.7$ & $<0.01$ & & 1.1 & $0.97-1.4$ & 0.11 \\
DFS stage II & 133 & 2.1 & $1.3-3.5$ & $<0.01$ & & 1.1 & $0.96-1.4$ & 0.15 \\
OS stage III & 94 & 2.8 & $1.8-4.4$ & $<0.01$ & & 1.1 & $0.98-1.3$ & 0.10 \\
DFS stage III & 92 & 2.3 & $1.0-4.9$ & 0.04 & & 1.1 & $0.83-1.3$ & 0.66
\end{tabular}

TIMP-1: tissue inhibitor of metalloproteinases 1; CEA: carcino-embryonic antigen; OS: overall survival; DFS: disease-free survival; HR: hazard ratio; and CI: confidence interval.

Currently, CEA is the only recommended preoperative serological biomarker in CRC, ${ }^{2}$ with a well-documented correlation with recurrence and survival. ${ }^{20-22} \mathrm{CEA}$ is used to subdivide stage II patients into prognostic groups of high- and low-risk patients, ${ }^{23}$ and is included in advanced nomograms for improving prognostic stratification of CRC patients. ${ }^{24} \mathrm{How}$ ever, CEA is unspecific and the sensitivity is considered insufficient for this purpose. ${ }^{25}$ Thus, there is still a need for additional biomarkers to be included in the daily management of patients with CRC. Some of the markers under evaluation include soluble uPAR variant forms, ${ }^{26} \mathrm{YKL}-40^{27}$ and miRNA. ${ }^{28}$ In addition, tissue markers, such as KRAS mutations and the presence of microsatellite instability, may be included in the near future as predictors of response to a particular treatment.

Plasma TIMP-1 has been suggested by EGTM as a promising prognostic biomarker. TIMP (1, 2, 3 and 4) regulate the activity of $\mathrm{MMP}^{4}$ enzymes involved in degradation of ECM and in cancer invasion and metastasis. Other specific functions of TIMP-1 are regulation of cell proliferation, ${ }^{29}$ 
Table 4 - Multivariate Cox regression analysis of all CRC patients (stages I-IV) for OS including the TIMP-1 and CEA levels and clinicopathological baseline variables.

\begin{tabular}{|c|c|c|c|c|}
\hline \multirow{2}{*}{$\begin{array}{l}\text { Parameter } \\
\text { Analysis of maximum likelihood estimate }\end{array}$} & \multirow[t]{2}{*}{$\operatorname{Pr}>\chi^{2}$} & \multirow[t]{2}{*}{ Hazard ratio } & \multicolumn{2}{|c|}{ 95\% Hazard ratio confidence limits } \\
\hline & & & & \\
\hline TIMP-1 (log base 2) & $<0.01$ & 1.8 & 1.3 & 2.4 \\
\hline Age (pr 10 year difference) & $<0.01$ & 1.4 & 1.1 & 1.7 \\
\hline Disease stage I versus IV & $<0.01$ & 0.06 & 0.03 & 0.14 \\
\hline Disease stage II versus IV & $<0.01$ & 0.06 & 0.03 & 0.12 \\
\hline Disease stage III versus IV & $<0.01$ & 0.19 & 0.11 & 0.31 \\
\hline Postoperative chemotherapy yes/no & 0.05 & 0.59 & 0.35 & 1.0 \\
\hline \multicolumn{5}{|l|}{ Removed } \\
\hline Localisation & 0.98 & & & \\
\hline Vascular invasion & 0.88 & & & \\
\hline Gender & 0.22 & & & \\
\hline Preoperative radiation therapy & 0.21 & & & \\
\hline CEA & 0.20 & & & \\
\hline Tumour differentiation grade & 0.12 & & & \\
\hline
\end{tabular}

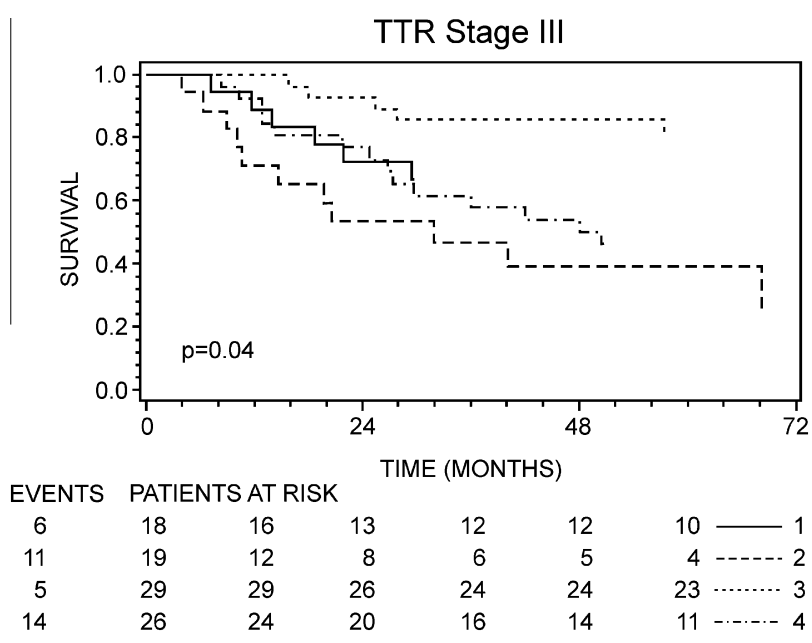

Fig. 3 - Kaplan-Meier estimates of time to recurrence in curatively treated stage III patients, stratified into four groups based on age- and gender-adjusted CEA and TIMP-1 levels, and whether adjuvant chemotherapy was given or not. Group 1: low TIMP-1 and without adjuvant chemotherapy; Group 2: high TIMP-1 level and without adjuvant chemotherapy; Group 3: low TIMP-1 level and with adjuvant chemotherapy and Group 4: high TIMP-1 level and with adjuvant chemotherapy. $P$-value is for the log-rank statistic. The number of patients at risk at $0,12,24,36,48$ and 60 months are shown below the axis and the number of events to the left. TIMP-1: tissue inhibitor of metalloproteinase 1; CEA: carcino-embryonic antigen and TTR: time to recurrence.

apoptosis $^{30}$ and angiogenesis. ${ }^{31}$ Most MMPs and TIMPs are not expressed by the cancer cells but are synthesised and secreted by adjacent stromal fibroblasts. ${ }^{32}$

Theoretically, TIMP-1 over-expression should inhibit tumour cell invasion and metastasis due to inhibition of MMP. Paradoxically, increased TIMP-1 expression is associated with negative prognosis in solid tumours. ${ }^{13,33}$ The increased expression of TIMP-1 may be related to the increased expres- sion of MMP during tumour progression; therefore, elevated levels of TIMP-1 in patients with invasive carcinomas may represent one of the host's responses to the remodelling stimuli through balancing local tissue degradation. ${ }^{4}$ It should be noted, however, that we have not been able to demonstrate a clear association between MMP and TIMP-1 expression levels in colorectal cancer. ${ }^{34}$ Increased TIMP-1 levels may in addition to MMP inhibition prevent apoptosis, thereby, improving cancer cell survival. It may also induce hepatocyte growth factor, which aids in the development of liver metastases. ${ }^{35}$ CRC is primarily diagnosed in the elderly population, although the range of ages at diagnosis is wide. Age together with disease stage is the strongest individual predictor of OS in CRC patients, but does not appear to influence the risk of recurrence. However, there are indirect effects of age: young patients have more advanced disease stage ${ }^{36}$ and older patients are less likely to be treated with adjuvant chemotherapy. ${ }^{37}$ This was reflected in the present cohort, where no patients 76 years or older and with stage III disease received chemotherapy (data not presented). However, there is a non-documented trend in Sweden since the 1990s that older patients receive chemotherapy based on estimation of biologic age and lack of co-morbidities. ${ }^{38}$

Age is particularly important in survival analysis for TIMP1 , as TIMP-1 levels increase with age and this can directly influence association with OS and DFS, and indirectly CSS and TTR, as older patients often do not obtain adjuvant treatment. Therefore, the analyses were corrected for age as a confounder by stratified and multivariate analyses: CEA had no association with age.

The difference in TTR in stage III patients stratified according to age- and gender-adjusted TIMP-1 and CEA levels and postoperative chemotherapy revealed major differences in recurrences, with only $17 \%$ developing recurrences in the group with low TIMP-1 and receiving adjuvant treatment, compared to $58 \%$ in the group with high TIMP-1 and no adjuvant treatment. Patients with both high and low values of TIMP-1 appeared to respond to postoperative chemotherapy (mainly 5FU and oxaliplatin in this cohort, data not shown). Although adjusted for age, the present cohort may be 
confounded through only younger patients ( $<75$ years) being offered adjuvant treatment.

TIMP-1 might be used as a prognostic marker identifying patients with poor prognosis and then offering these high TIMP-1 patients additional treatment. An example of this is seen in the stage III patients in this cohort (Fig. 3) where patients with high TIMP-1 levels receiving no chemotherapy have the highest risk of recurrence. Of specific interest is that stage III-high TIMP-1 patients appear to receive benefit from adjuvant chemotherapy, i.e. high TIMP-1 patients seem to be responsive to adjuvant chemotherapy. However, it should be kept in mind that the results may be confounded by the fact that older individuals have higher TIMP-1 and are less likely to receive chemotherapy at the time of the study.

In conclusion, this independent prospective validation study confirms the significant association between preoperative plasma TIMP-1 levels and survival of CRC patients: TIMP1 provided stronger prognostic information than CEA. Thus, this validation study confirms initial observations on plasma TIMP-1 as a stage independent prognostic marker for CRC patients and thus brings plasma TIMP-1 to Level of Evidence II, according to the Tumour Marker Utility Grading System for tumour marker evaluation described by Hayes and colleagues. $^{39}$

\section{Conflict of interest statement}

None declared.

\section{Acknowledgements}

Research nurse Eva Strand, for obtaining informed consent from the patients, and docent Kennet Smedh, Head of the Colorectal section for supporting this study at the clinic, both work at the Department of Surgery, Central District Hospital, Västerås, Sweden. This study was funded by the research fund of the county of Västmanland against cancer, Lions Cancer Foundation Uppsala, the Swedish Cancer Society, the Danish Cancer Society, the Danish Strategic Research Council and the Redaktoer Kaaresens Foundation. Vibeke Jensen and Birgitte Sander Nielsen are thanked for the excellent work in analysing the samples.

\section{R E F E R E N C E S}

1. Ferlay J, Parkin DM, Steliarova-Foucher E. Estimates of cancer incidence and mortality in Europe in 2008. Eur J Cancer 2010;46(4):765-81.

2. Locker GY, Hamilton S, Harris J, et al. ASCO 2006 update of recommendations for the use of tumor markers in gastrointestinal cancer. J Clin Oncol 2006;24(33):5313-27.

3. Duffy MJ, van Dalen A, Haglund C, et al. Tumour markers in colorectal cancer: European Group on Tumour Markers (EGTM) guidelines for clinical use. Eur J Cancer 2007;43(9):1348-60.

4. Jiang Y, Goldberg ID, Shi YE. Complex roles of tissue inhibitors of metalloproteinases in cancer. Oncogene 2002;21(14):2245-52.
5. Hewitt RE, Brown KE, Corcoran M, Stetler-Stevenson WG. Increased expression of tissue inhibitor of metalloproteinases type 1 (TIMP-1) in a more tumourigenic colon cancer cell line. J Pathol 2000;192(4):455-9.

6. Holten-Andersen MN, Christensen IJ, Nielsen HJ, et al. Total levels of tissue inhibitor of metalloproteinases 1 in plasma yield high diagnostic sensitivity and specificity in patients with colon cancer. Clin Cancer Res 2002;8(1):156-64.

7. Caspersen MB, Sorensen NM, Schrohl AS, et al. Investigation of tissue inhibitor of metalloproteinases 1 in plasma from colorectal cancer patients and blood donors by surfaceenhanced laser desorption/ionization time-of-flight mass spectrometry. Int J Biol Markers 2007;22(2):89-94.

8. Holten-Andersen MN, Fenger C, Nielsen HJ, et al. Plasma TIMP-1 in patients with colorectal adenomas: a prospective study. Eur J Cancer 2004;40(14):2159-64.

9. Holten-Andersen $\mathrm{M}$, Christensen IJ, Nilbert M, et al. Association between preoperative plasma levels of tissue inhibitor of metalloproteinases 1 and rectal cancer patient survival: a validation study. Eur J Cancer 2004;40(1):64-72.

10. Holten-Andersen MN, Stephens RW, Nielsen HJ, et al. High preoperative plasma tissue inhibitor of metalloproteinase-1 levels are associated with short survival of patients with colorectal cancer. Clin Cancer Res 2000;6(11):4292-9.

11. Holten-Andersen MN, Nielsen HJ, Sorensen S, et al. Tissue inhibitor of metalloproteinases-1 in the postoperative monitoring of colorectal cancer. Eur J Cancer 2006;42(12):1889-96.

12. Giaginis C, Nikiteas N, Margeli A, et al. Serum tissue inhibitor of metalloproteinase 1 and 2 (TIMP-1 and TIMP-2) levels in colorectal cancer patients: associations with clinicopathological variables and patient survival. Int J Biol Markers 2009;24(4):245-52.

13. Waas ET, Hendriks T, Lomme RM, Wobbes T. Plasma levels of matrix metalloproteinase-2 and tissue inhibitor of metalloproteinase-1 correlate with disease stage and survival in colorectal cancer patients. Dis Colon Rectum 2005;48(4):700-10.

14. Yukawa $\mathrm{N}$, Yoshikawa $\mathrm{T}$, Akaike $\mathrm{M}$, et al. Impact of plasma tissue inhibitor of matrix metalloproteinase-1 on long-term survival in patients with colorectal cancer. Oncology 2007;72(3-4):205-8.

15. Holten-Andersen MN, Murphy G, Nielsen HJ, et al. Quantitation of TIMP-1 in plasma of healthy blood donors and patients with advanced cancer. Brit J Cancer 1999;80(3-4): 495-503.

16. Punt CJ, Buyse $\mathrm{M}$, Kohne $\mathrm{CH}$, et al. Endpoints in adjuvant treatment trials: a systematic review of the literature in colon cancer and proposed definitions for future trials. J Natl Cancer Inst 2007;99(13):998-1003.

17. McShane LM, Altman DG, Sauerbrei W, et al. REporting recommendations for tumor MARKer prognostic studies (REMARK). Breast Cancer Res Treat 2006;100(2):229-35.

18. Sorensen NM, Bystrom P, Christensen IJ, et al. TIMP-1 is significantly associated with objective response and survival in metastatic colorectal cancer patients receiving combination of irinotecan, 5-fluorouracil, and folinic acid. Clin Cancer Res 2007;13(14):4117-22.

19. AJCC (American Joint Committee on Cancer). Staging of colon and rectal cancer; 2002.

20. Wanebo HJ, Rao B, Pinsky CM, et al. Preoperative carcinoembryonic antigen level as a prognostic indicator in colorectal cancer. New Engl J Med 1978;299(9):448-51.

21. Sener SF, Imperato JP, Chmiel J, Fremgen A, Sylvester J. The use of cancer registry data to study preoperative carcinoembryonic antigen level as an indicator of survival in colorectal cancer. CA Cancer J Clin 1989;39(1):50-7. 
22. Park YJ, Park KJ, Park JG, et al. Prognostic factors in 2230 Korean colorectal cancer patients: analysis of consecutively operated cases. World J Surg 1999;23(7):721-6.

23. Quah HM, Chou JF, Gonen M, et al. Identification of patients with high-risk stage II colon cancer for adjuvant therapy. Dis Colon Rectum 2008;51(5):503-7.

24. Weiser MR, Landmann RG, Kattan MW, et al. Individualized prediction of colon cancer recurrence using a nomogram. J Clin Oncol 2008;26(3):380-5.

25. Hara M, Kanemitsu Y, Hirai T, Komori K, Kato T. Negative serum carcinoembryonic antigen has insufficient accuracy for excluding recurrence from patients with Dukes C colorectal cancer: analysis with likelihood ratio and posttest probability in a follow-up study. Dis Colon Rectum 2008;51(11):1675-80.

26. Seetoo DQ, Crowe PJ, Russell PJ, Yang JL. Quantitative expression of protein markers of plasminogen activation system in prognosis of colorectal cancer. J Surg Oncol 2003;82(3):184-93.

27. Cintin C, Johansen JS, Christensen IJ, et al. High serum YKL40 level after surgery for colorectal carcinoma is related to short survival. Cancer 2002;95(2):267-74.

28. Faber C, Kirchner T, Hlubek F. The impact of microRNAs on colorectal cancer. Virchows Arch 2009;454(4):359-67.

29. Rho SB, Chung BM, Lee JH. TIMP-1 regulates cell proliferation by interacting with the ninth zinc finger domain of PLZF. J Cell Biochem 2007;101(1):57-67.

30. Guedez L, Stetler-Stevenson WG, Wolff L, et al. In vitro suppression of programmed cell death of B cells by tissue inhibitor of metalloproteinases-1. J Clin Invest 1998;102(11):2002-10.

31. Ohtani Y, Aoe M, Hara F, et al. Suppression effects of human recombinant tissue inhibitor of metalloproteinases-1 (TIMP-1) on tumor proliferation using in vivo treatment model of welldifferentiated colon cancer cell line, HT29. Acta Med Okayama 2006;60(5):257-66.

32. Holten-Andersen MN, Hansen U, Brunner N, et al. Localization of tissue inhibitor of metalloproteinases 1 (TIMP1) in human colorectal adenoma and adenocarcinoma. Int $J$ Cancer 2005;113(2):198-206.

33. Sorensen NM, Sorensen IV, Wurtz SO, et al. Biology and potential clinical implications of tissue inhibitor of metalloproteinases-1 in colorectal cancer treatment. Scand $J$ Gastroenterol 2008;43(7):774-86.

34. Offenberg H, Brunner N, Mansilla F, Orntoft Torben F, Birkenkamp-Demtroder K. TIMP-1 expression in human colorectal cancer is associated with TGF-B1, LOXL2, INHBA1, TNF-AIP6 and TIMP-2 transcript profiles. Mol Oncol 2008;2(3):233-40.

35. Kopitz C, Gerg M, Bandapalli OR, et al. Tissue inhibitor of metalloproteinases-1 promotes liver metastasis by induction of hepatocyte growth factor signaling. Cancer Res 2007;67(18):8615-23.

36. Ries LAG, Kosary CL, Hankey BF, et al. SEER cancer statistics review, 1973-1999. Bethesda, MD: National Cancer Institute; 2002. <http://seer.cancer.gov/>.

37. Lemmens V, Steenbergen LV, Janssen-Heijnen M, et al.. Trends in colorectal cancer in the south of the Netherlands 19752007: rectal cancer survival levels with colon cancer survival. Acta Oncol 2010;April 29 [Epub ahead of print].

38. Papamichael D, Audisio R, Horiot JC, et al. Treatment of the elderly colorectal cancer patient: SIOG expert recommendations. Ann Oncol 2009;20(1):5-16.

39. Hayes DF, Bast RC, Desch CE, et al. Tumor marker utility grading system: a framework to evaluate clinical utility of tumor markers. J Natl Cancer Inst 1996;88(20):1456-66. 